IJMMS 27:12 (2001) 737-747

PII. S0161171201005841

http://ijmms.hindawi.com

(C) Hindawi Publishing Corp.

\title{
AN INTERARRIVAL HYPEREXPONENTIAL MACHINE INTERFERENCE WITH BALKING, RENEGING, STATE-DEPENDENT, SPARES, AND AN ADDITIONAL SERVER FOR LONGER QUEUES
}

\author{
RAGAB OMARAH AL-SEEDY and FAWZIAH M. AL-IBRAHEEM
}

(Received 16 March 1999 and in revised form 1 March 2000)

\begin{abstract}
The aim of this paper is to treat the analytical solution of the truncated interarrival hyperexponential machine interference queue: $H_{2} / M / 1 / m+Y / m+Y$ in case of two branches with the following concepts: balking, reneging, state-dependent, spares, and an additional server for longer queues. Our research treats the general case for the values of $m$ and $Y$ considering the discipline FIFO. And some special cases have been verified.
\end{abstract}

2000 Mathematics Subject Classification. 60K25.

1. Introduction. The truncated interarrival hyperexponential queue $H_{2} / \mathrm{M} / 1 / \mathrm{m}+$ $y / m+y$ (in case of two branches) treated numerically by Gupta [6]. Al-Seedy [2] treated analytically in some cases of $Y=0$ and $m=1,2,3$. Also Al-Seedy [3] studied the general case of $m$ but with $Y=0$ with the two concepts of balking and reneging. Abou-El-Ata [1] treated the analytical solution of the truncated interarrival hyperexponential machine interference queue $H_{2} / \mathrm{M} / \mathrm{C} / \mathrm{m} / \mathrm{m}$ with both balking and reneging concepts only.

In this paper, we treat the analytical solution of the truncated interarrival hyperexponential machine interference queue $H_{2} / M / 1 / m+Y / m+Y$ with the concepts of balking, reneging, state-dependent, spares, and an additional server for longer queues. The discipline considered is FIFO.

2. Analyzing the problem. Consider the two-channels truncated interarrival hyperexponential queue having two branches with rates $\alpha \lambda_{1}$ and $(1-\alpha) \lambda_{2}$, and the service time is an exponential with service rate $\mu$.

Assume the balking concept with probability

$$
\beta=\operatorname{Prob}\{\text { a unit joins the queue }\}
$$

where

$$
0 \leq \beta<1 \quad \text { if } n=1(1) \overline{m+Y}, \quad \beta=1 \quad \text { if } n=0 .
$$

Also, the reneging concept in case of $n$ units with probability is

$$
r(n)=\operatorname{Prob}\{\text { a unit leaves the queue }\}=(n-1) \delta,
$$


where

$$
r(n)=0 \text { for } n=0,1, \quad 0<r(n) \leq 1 \quad \text { for } n=2(1) \overline{m+Y} \text {, }
$$

and $\delta$ is the rate of time $t$, having the probability density function $f(t)=\delta e^{-\delta t}$.

The interarrival rates $\lambda_{n}$ in case of machine with spares are

$$
\lambda_{n}= \begin{cases}m \lambda, & n=0, \\ m \beta \lambda, & 1 \leq n \leq Y, \\ (m-n+y) \beta \lambda, & Y<n<Y+m, \\ 0, & n \geq Y+m .\end{cases}
$$

But the service time rates in case of the concepts of state-dependent and an additional server for longer queues are

$$
\mu_{n}= \begin{cases}\mu_{1}, & 0 \leq n \leq k_{1}, \\ \mu_{2}, & k_{1} \leq n \leq k_{2}, \\ \mu_{2}+\mu_{3}=\mu, & k_{2} \leq n \leq m+Y .\end{cases}
$$

Define the probabilities:

$P_{n, j}=\operatorname{Prob}\{n$ units in the system and $j$ the arrival branch occupied by the next arrival unit\} where $n \geq 0$ and $j=1,2$.

The steady-state difference equations of the queue $H_{2} / M / 1 / m+Y / m+Y$ considering all the concepts of balking, reneging, state-dependent, spares, and an additional server for longer queues, are

- for $n=0$,

$$
\begin{aligned}
& m \lambda_{1} P_{0,1}=\mu_{1} P_{1,1}, \\
& m \lambda_{2} P_{0,2}=\mu_{1} P_{1,2},
\end{aligned}
$$

- for $n=1$,

$$
\begin{aligned}
& {\left[m \beta \lambda_{1}+\mu_{1}\right] P_{1,1}=\alpha m \lambda_{1} P_{0,1}+\alpha m \lambda_{2} P_{0,2}+\left(\mu_{1}+\delta\right) P_{2,1},} \\
& {\left[m \beta \lambda_{2}+\mu_{1}\right] P_{1,2}=(1-\alpha) m \lambda_{1} P_{0,1}+(1-\alpha) m \lambda_{2} P_{0,2}+\left(\mu_{1}+\delta\right) P_{2,2},}
\end{aligned}
$$

- for $2 \leq n \leq Y$,

$$
\begin{aligned}
& {\left[m \beta \lambda_{1}+\mu_{1}+(n-1) \delta\right] P_{n, 1}} \\
& \quad=\alpha m \beta \lambda_{1} P_{n-1,1}+\alpha m \beta \lambda_{2} P_{n-1,2}+\left(\mu_{1}+n \delta\right) P_{n+1,1}, \\
& {\left[m \beta \lambda_{2}+\mu_{1}+(n-1) \delta\right] P_{n, 2}} \\
& \quad=(1-\alpha) m \beta \lambda_{1} P_{n-1,1}+(1+\alpha) m \beta \lambda_{2} P_{n-1,2}+\left(\mu_{1}+n \delta\right) P_{n+1,2},
\end{aligned}
$$


- for $Y<n<k_{1}-1$,

$$
\begin{aligned}
{[(m-n} & \left.+Y) \beta \lambda_{1}+\mu_{1}+(n-1) \delta\right] P_{n, 1} \\
= & \alpha(m-n+Y+1) \beta \lambda_{1} P_{n-1,1}+\alpha(m-n+Y+1) \beta \lambda_{2} P_{n-1,2} \\
& +\left(\mu_{1}+n \delta\right) P_{n+1,1}, \\
{[(m-n} & \left.+Y) \beta \lambda_{2}+\mu_{1}+(n-1) \delta\right] P_{n, 2} \\
= & (1-\alpha)(m-n+Y+1) \beta \lambda_{1} P_{n-1,1}+(1-\alpha)(m-n+Y+1) \beta \lambda_{2} P_{n-1,2} \\
& +\left(\mu_{1}+n \delta\right) P_{n+1,2},
\end{aligned}
$$

- for $n=k_{1}-1$,

$$
\begin{aligned}
{\left[\left(m-k_{1}\right.\right.} & \left.+Y+1) \beta \lambda_{1}+\mu_{1}+\left(k_{1}-2\right) \delta\right] P_{K_{1}-1,1} \\
= & \alpha\left(m-k_{1}+Y+2\right) \beta \lambda_{1} P_{k_{1}-2,1}+\alpha\left(m-k_{1}+Y+2\right) \beta \lambda_{2} P_{k_{1}-2,2} \\
& +\left[\mu_{2}+\left(k_{1}-1\right) \delta\right] P_{k_{1}, 1}, \\
{[(m-} & \left.\left.k_{1}+Y+1\right) \beta \lambda_{2}+\mu_{1}+\left(k_{1}-2\right) \delta\right] P_{k_{1}-1,2} \\
= & (1-\alpha)\left(m-k_{1}+Y+2\right) \beta \lambda_{1} P_{k_{1}-2,1}+(1-\alpha)\left(m-k_{1}+Y+2\right) \beta \lambda_{2} P_{k_{1}-2,2} \\
& +\left[\mu_{2}+\left(k_{1}-1\right) \delta\right] P_{k_{1}, 2},
\end{aligned}
$$

- for $k_{1} \leq n<k_{2}-1$,

$$
\begin{aligned}
{[(m-n} & \left.+Y) \beta \lambda_{1}+\mu_{2}+(n-1) \delta\right] P_{n, 1} \\
= & \alpha(m-n+Y+1) \beta \lambda_{1} P_{n-1,1}+\alpha(m-n+Y+1) \beta \lambda_{2} P_{n-1,2} \\
& +\left(\mu_{2}+n \delta\right) P_{n+1,1} \\
{[(m-n} & \left.+Y) \beta \lambda_{2}+\mu_{2}+(n-1) \delta\right] P_{n, 2} \\
= & (1-\alpha)(m-n+Y+1) \beta \lambda_{1} P_{n-1,1} \\
& +(1-\alpha)(m-n+Y+1) \beta \lambda_{2} P_{n-1,2}+\left(\mu_{2}+n \delta\right) P_{n+1,2},
\end{aligned}
$$

- for $n=k_{2}-1$,

$$
\begin{aligned}
{\left[\left(m-k_{2}\right.\right.} & \left.+Y+1) \beta \lambda_{1}+\mu_{2}+\left(k_{2}-2\right) \delta\right] P_{K_{2}-1,1} \\
= & \alpha\left(m-k_{2}+Y+2\right) \beta \lambda_{1} P_{k_{2}-2,1}+\alpha\left(m-k_{2}+Y+2\right) \beta \lambda_{2} P_{k_{2}-2,2} \\
& +\left(\mu+\left(k_{2}-1\right) \delta\right) P_{k_{2}, 1}, \\
{\left[\left(m-k_{2}\right.\right.} & \left.+Y+1) \beta \lambda_{2}+\mu_{2}+\left(k_{2}-2\right) \delta\right] P_{k_{2}-1,2} \\
= & (1-\alpha)\left(m-k_{2}+Y+2\right) \beta \lambda_{1} P_{k_{2}-2,1}+(1-\alpha)\left(m-k_{2}+Y+2\right) \beta \lambda_{2} P_{k_{2}-2,2} \\
& +\left(\mu+\left(k_{2}-1\right) \delta\right) P_{k_{2}, 2},
\end{aligned}
$$


- for $k_{2} \leq n<m+Y$,

$$
\begin{aligned}
{[(m-n} & \left.+Y) \beta \lambda_{1}+\mu+(n-1) \delta\right] P_{n 1} \\
= & \alpha(m-n+Y+1) \beta \lambda_{1} P_{n-1,1}+\alpha(m-n+Y+1) \beta \lambda_{2} P_{n-1,2} \\
& +(\mu+n \delta) P_{n+1,1} \\
{[(m-n} & \left.+Y) \beta \lambda_{2}+\mu+(n-1) \delta\right] P_{n 2} \\
= & (1-\alpha)(m-n+Y+1) \beta \lambda_{1} P_{n-1,1} \\
& +(1-\alpha)(m-n+Y+1) \beta \lambda_{2} P_{n-1,2}+(\mu+n \delta) P_{n+1,2},
\end{aligned}
$$

- for $n=m+Y$,

$$
\begin{aligned}
& {[\mu+(m+Y-1) \delta] P_{m+Y, 1}=\alpha \beta \lambda_{1} P_{m+Y-1,1}+\alpha \beta \lambda_{2} P_{m+Y-1,2}} \\
& {[\mu+(m+Y-1) \delta] P_{m+Y, 2}=(1-\alpha) \beta \lambda_{1} P_{m+Y-1,1}+(1-\alpha) \beta \lambda_{2} P_{m+Y-1,2}}
\end{aligned}
$$

Write

$$
\rho_{i}=\frac{\lambda_{1}}{\mu_{1}}, \quad \rho_{i}^{*}=\frac{\lambda_{1}}{\mu_{2}}, \quad \bar{\rho}_{i}=\frac{\lambda_{1}}{\mu}, \quad \gamma=\frac{\delta}{\mu_{1}}, \quad \gamma^{*}=\frac{\delta}{\mu_{2}}, \quad \bar{\gamma}=\frac{\delta}{\mu}, \quad i=1,2 .
$$

And add every two equations in each step to simplify the required solution. We find

$$
\begin{aligned}
& m \rho_{1} P_{0,1}=P_{1,1}, \quad n=0, \\
& \left(m B \rho_{1}+1\right) P_{1,1}+\left(m B \rho_{2}+1\right) P_{1,2} \\
& =(1+\gamma) P_{2,2}+m \rho_{1} P_{0,1}+m \rho_{2} P_{0,2}+(\gamma+1) P_{2,1}, \quad n=1, \\
& {\left[\left(m B \rho_{1}+1+(n-1) \gamma\right)\right] P_{n, 1}+\left[\left(m B \rho_{2}+1+(n-1) \gamma\right)\right] P_{n, 2}} \\
& =m B \rho_{1} P_{n-1,1}+(1+n \gamma) P_{n+1,1}+m B \rho_{2} P_{n-1,2+(1+n \gamma) P_{n+1,2}}, \quad 2 \leq n \leq Y, \\
& {\left[(m-n+Y) B \rho_{1}+1+(n-1) \gamma\right] P_{n, 1}+\left[(m-n+Y) B \rho_{2}+1+(n-1) \gamma\right] P_{n, 2}} \\
& =(m-n+Y+1) B \rho_{1} P_{n-1,1}+(m-n+Y+1) B \rho_{2} P_{n-1,2} \\
& +(1+n \gamma) P_{n+1,1}+(1+n \gamma) P_{n+1,2}, \quad Y<n<k_{1}-1, \\
& {\left[\left(m-k_{1}+1+Y\right) B \rho_{1}^{*}+\frac{\mu_{1}}{\mu_{2}}+\left(k_{1}-2\right) \gamma^{*}\right] P_{k_{1}-1,1}} \\
& +\left[\left(m-k_{1}+1+Y\right) B \rho_{2}^{*}+\frac{\mu_{1}}{\mu_{2}}+\left(k_{1}-2\right) \gamma^{*}\right] P_{k_{1}-1,2} \\
& =\left(m-k_{1}+2+Y\right) B \rho_{2}^{*} P_{k_{1}-2,2}+\left(1+\left(k_{1}-1\right) \gamma^{*}\right) P_{k_{1}, 1} \\
& +\left(1+\left(k_{1}-1\right) \gamma^{*}\right) P_{k_{1}, 2}+\left(m-k_{1}+2+Y\right) B \rho_{1}^{*} P_{k_{1}-2,1}, \quad n=k_{1}-1,
\end{aligned}
$$




$$
\begin{aligned}
{[(m-} & \left.n+Y) B \rho_{1}^{*}+1+(n-1) \delta^{*}\right] P_{n, 1}+\left[(m-n+Y) B \rho_{2}^{*}+1+(n-1) \gamma^{*}\right] P_{n, 2} \\
= & (m-n+Y+1) B \rho_{1}^{*} P_{n-1,1}+\left(1+n \gamma^{*}\right) P_{n+1,2} \\
& +(m-n+Y+1) B \rho_{2}^{*} P_{n-1,2}+\left(1+n \gamma^{*}\right) P_{n+1,1}, \quad k_{1} \leq n<k_{2}-1, \\
{[(m-} & \left.\left.k_{2}+1+Y\right) B \bar{\rho}_{1}+\frac{\mu_{2}}{\mu}+\left(k_{2}-2\right) \bar{\gamma}\right] P_{k_{2}-1,1} \\
& +\left[\left(m-k_{2}+1+Y\right) B \bar{\rho}_{2}+\frac{\mu_{2}}{\mu}+\left(k_{2}-2\right) \bar{\gamma}\right] P_{k_{2}-1,2} \\
= & \left(m-k_{2}+2+Y\right) B \bar{\rho}_{1} P_{k_{1}-2,1}+\left(m-k_{2}+2+Y\right) B \bar{\rho}_{2} P_{k_{2}-2,2} \\
& +\left(1+\left(k_{2}-1\right) \bar{\gamma}\right) P_{k_{2}, 1}+\left(1+\left(k_{2}-1\right) \bar{\gamma}\right) P_{k_{2}, 2}, \quad n=k_{2}-1, \\
{[(m-} & \left.n+Y) B \bar{\rho}_{1}+1+(n-1) \bar{\gamma}\right] P_{n, 1}+\left[(m-n+Y) B \bar{\rho}_{2}+1+(n-1) \bar{\gamma}\right] P_{n, 2} \\
= & (m-n+Y+1) B \bar{\rho}_{1} P_{n-1,1}+(m-n+Y+1) B \bar{\rho}_{2} P_{n-1,2} \\
& +(1+n \bar{\gamma}) P_{n+1,1}+(1+n \bar{\gamma}) P_{n+1,2}, \quad k_{2} \leq n<m+Y, \\
{[1+} & (m+y-1) \bar{\gamma}] P_{m+Y, 1}+[1+(m+Y-1) \bar{\gamma}] P_{m+Y, 2} \\
= & B \bar{\rho}_{1} P_{m+Y-1,1}+B \bar{\rho}_{2} P_{m+Y-1,2}, \quad n=Y+m .
\end{aligned}
$$

These are $(m+Y+1)$ equations in the unknowns $P_{n, j}$. To solve them for $P_{n, j}$, we need the formula which gives the sum of the probabilities at every branch. We introduce the formula in the following lemma.

LEMMA 2.1. For the truncated hyperexponential machine interference queue $\mathrm{H}_{2} / \mathrm{M} /$ $1 / m+Y / m+Y$ with the concepts of balking, reneging, state-dependent, spares, and an additional server for longer queues, there is

$$
\begin{aligned}
\sum_{n=0}^{m+Y} P_{n, 1}= & \frac{1}{m B\left\{\alpha \lambda_{2}+(1-\alpha) \lambda_{1}\right\}} \\
\times & {\left[\alpha m \lambda_{2}\left\{\beta+(1-\beta) P_{0,2}\right\}+\alpha \beta \lambda_{2} Y \sum_{n=Y}^{m+Y} P_{n, 2}\right.} \\
& -m \lambda_{1}(1-\beta)(1-\alpha) P_{0,1}-\alpha \beta \lambda_{2} \sum_{n=Y}^{m+Y} n P_{n, 2} \\
& \left.+\beta \lambda_{1}(1-\alpha) \sum_{n=Y}^{m+Y} n P_{n, 1}-\beta \lambda_{1} Y(1-\alpha) \sum_{n=Y}^{m+Y} P_{n, 1}\right]=\eta, \\
& \sum_{n=0}^{m+Y} P_{n, 2}=1-\eta .
\end{aligned}
$$


Proof. Adding either the nine first relations or the nine second relations, we get

$$
\begin{aligned}
& m \beta \lambda_{1}(1-\alpha) \sum_{n=0}^{m+Y} P_{n, 1}-\alpha m \beta \lambda_{2} \sum_{n=0}^{m+Y} P_{n, 2} \\
& =\alpha m \lambda_{2}(1-\beta) P_{0,2}-m \lambda_{1}(1-\beta)(1-\alpha) P_{0,1}+\alpha \beta \lambda_{2} Y \sum_{n=Y}^{m+Y} P_{n, 2}-\alpha \beta \lambda_{2} \sum_{n=Y}^{m+Y} n P_{n, 2} \\
& \quad+\beta \lambda_{1}(1-\alpha) \sum_{n=Y}^{m+Y} n P_{n, 1}-\beta \lambda_{1} Y(1-\alpha) \sum_{n=Y}^{m+Y} P_{n, 1} .
\end{aligned}
$$

But

$$
\sum_{n=Y}^{m+Y}\left(P_{n, 1}+P_{n, 2}\right)=1 .
$$

Multiply (2.20) by $\alpha m \lambda_{2} B$ then add to (2.19), we obtain relation (2.18), that is, the concepts of reneging, state-dependent, and an additional server for longer queues are not affecting the results of the lemma.

Now to solve the set of equations (2.17), first we need to solve $P_{n, 1}$ (the first branch probabilities) in terms of $P_{n, 2}$ (the second branch probabilities) by the following method:

We arrange the coefficients of equations (2.17) in a square matrix of order $(m+$ $Y+1$ ) after replacing (2.18) instead of the last equation (2.17). By doing elementary row-operations. We get the following solution's formula:

From the last row $i=1$, we get

$$
P_{m+Y, 1}=\frac{\alpha}{1-\alpha} P_{m+Y, 2}=A, \quad n=m+Y,
$$

where $i=m+Y+1-n$.

At $i=2$,

$$
\begin{gathered}
B \bar{\rho}_{1} P_{m+Y-1,1}-[1+(m+Y-1) \bar{\gamma}] P_{m+Y, 1}=[1+(m+Y-1) \bar{\gamma}] P_{m+Y, 2}-B \bar{\rho}_{1} P_{m+Y-1,2}, \\
B \bar{\rho}_{1} P_{m+Y-1,1}-[1+(m+Y-1) \bar{\gamma}] A+[1+(m+Y-1) \bar{\gamma}] P_{m+Y, 2}-B \bar{\rho}_{1} P_{m+Y-1,2}=B, \\
P_{m+Y-1,1}=\frac{B}{\beta \bar{\rho}_{1}}, \quad n=m+Y-1 .
\end{gathered}
$$

At $i=3$,

$$
\begin{aligned}
P_{m+Y-2,1}=\frac{1}{2 \beta \rho_{1}}[ & \{1+(m+Y-2) \bar{\gamma}\} P_{m+Y-1,2} \\
& \left.-2 \beta \bar{\rho}_{2} P_{m+Y-2,2}+\frac{1+(m+Y-2) \bar{\gamma}}{\beta \bar{\rho}_{1}} B\right], \quad n=m+Y-2 .
\end{aligned}
$$

And so on. 
Then we can find the following formula for the solutions to the rows $4 \leq i \leq m+$ $Y-\left(k_{2}-2\right)$, that is, $k_{2}-1 \leq n \leq m+Y-3$ :

$$
\begin{aligned}
p_{n, 1}= & \frac{1}{(m+Y-n) B \bar{\rho}_{1}} \\
\times & {\left[B \frac{(1+n \bar{\gamma})_{(m+Y-n-1) \bar{\gamma}}}{(m+Y-n-1) !\left(B \bar{\rho}_{1}\right)^{(m+Y-n-1)}}\right.} \\
& \quad B \bar{\rho}_{2} \sum_{r=1}^{m+Y-n-1} \frac{(r+1)(1+n \gamma)_{(m+Y-n-r-1) \bar{\gamma}}}{[m+Y-n-1]_{(m+Y-n-r-1)}\left(B \bar{\rho}_{1}\right)^{(m+Y-n-r-1)}} P_{(m+Y-r-1,2)} \\
& \left.+\sum_{r=1}^{m+Y-n-1} \frac{(1+n \gamma)_{(m+Y-n-r-1) \bar{\gamma}}}{[m+Y-n-1]_{(m+Y-n-r-1)}\left(B \bar{\rho}_{1}\right)^{(m+Y-n-r-1)}} P_{(m+Y-r, 2)}\right], \\
& k_{2}-1 \leq n \leq m+Y-3 .
\end{aligned}
$$

In particular for $n=k_{2}-1$, we find the value of $P_{k_{2}-1,1}$

$$
\begin{aligned}
&\left(m-k_{2}+Y+1\right) B \bar{\rho}_{1} P_{k_{2}-1,1} \\
&=T_{1}=B \frac{\left(1+\left(k_{2}-1\right) \bar{\gamma}\right)_{\left(m+Y-k_{2}\right) \bar{\gamma}}}{\left(m+Y-k_{2}\right) !\left(B \bar{\rho}_{1}\right)^{\left(m+Y-k_{2}\right)}} \\
&-B \bar{\rho}_{2} \sum_{r=1}^{m+Y-k_{2}} \frac{(r+1)\left(1+\left(k_{2}-1\right) \bar{\gamma}\right)_{\left(m+Y-k_{2}-r\right) \bar{\gamma}}}{\left[m+Y-k_{2}\right]_{\left(m+Y-k_{2}-r\right)}\left(B \bar{\rho}_{1}\right)^{\left(m+Y-k_{2}-r\right)}} P_{(m+Y-r-1,2)} \\
&+\sum_{r=1}^{m+Y-k_{2}} \frac{\left(1+\left(k_{2}-1\right) \bar{\gamma}\right)_{\left(m+Y-k_{2}-r+1\right) \bar{\gamma}}}{\left[m+Y-k_{2}\right]_{\left(m+Y-k_{2}-r\right)}\left(B \bar{\rho}_{1}\right)^{\left(m+Y-k_{2}-r\right)}} P_{(m+Y-r, 2)} .
\end{aligned}
$$

Now from row $i=m+Y-\left(k_{2}-3\right)$, the value of $P_{k_{2}-2,1}$ is

$$
\begin{aligned}
p_{k_{2}-2,1}\left(m-k_{1}+Y+2\right) B \rho_{1}^{*}= & \left\{1+\left(k_{2}-2\right) \gamma^{*}\right\} P_{k_{2}-1,2}-\left(m-k_{2}+Y+2\right) B \rho_{2}^{*} P_{k_{2}-2,2} \\
& +T_{1} \frac{1+\left(k_{2}-2\right) \gamma^{*}}{\left(m-k_{2}+Y+1\right) B \rho_{1}}=D
\end{aligned}
$$

Then

$$
P_{k_{2}-2,1}=\frac{D}{\left(m-k_{1}+Y+2\right) B \rho_{1}^{*}} .
$$

From the row $i=m+Y-\left(k_{2}-4\right)$, the value of $P_{k_{2}-3,1}$ is

$$
\begin{aligned}
P_{k_{2}-3,1}=\frac{1}{\left(m-k_{2}+Y+3\right) B \rho_{1}^{*}}[ & \left\{1+\left(k_{2}-3\right) \gamma^{*}\right\} P_{k_{2}-2,2}-\left(m-k_{2}+Y+3\right) B \rho_{2}^{*} P_{k_{2}-3,2} \\
& \left.+\frac{1+\left(k_{2}-3\right) \gamma^{*}}{\left(m-k_{2}+Y+2\right) B \rho_{1}^{*}} D\right]
\end{aligned}
$$


then we can obtain the formula for the solutions to the rows $m+Y-\left(k_{2}-5\right) \leq i \leq$ $m+Y-\left(k_{1}-2\right)$, that is, $k_{1}-1 \leq n \leq k_{2}-4$ as

$$
\begin{aligned}
P_{n, 1}= & \frac{1}{(m+Y-n) B \rho_{1}^{*}} \\
\times & \quad\left[\frac{\left(1+n \gamma^{*}\right)_{\left(k_{1}-n-2\right) \gamma^{*}}}{[m+Y-n-1]_{\left(k_{2}-n-2\right)}\left(B \rho_{1}^{*}\right)^{\left(k_{2}-n-2\right)}}\right. \\
& \quad-B \rho_{2}^{*} \sum_{r-1}^{k_{2}-n-2} \frac{\left(m-k_{2}+Y+r+2\right)\left(1+n \gamma^{*}\right)_{\left(k_{2}-n-r-2\right) \gamma^{*}}}{[m+Y-n-1]_{\left(k_{2}-n-r-2\right)}\left(B \rho_{1}\right)^{\left(k_{2}-n-r-2\right)}} P_{k_{2}-r-2,2} \\
& \left.\quad+\sum_{r-1}^{k_{2}-n-2} \frac{\left(1+n \gamma^{*}\right)_{\left(k_{1}-n-r-1\right) \gamma^{*}}}{[m+Y-n-1]_{\left(k_{2}-n-r-2\right)}\left(B \rho_{1}^{*}\right)^{\left(k_{2}-n-r-2\right)}} P_{k_{2}-r-1,2}\right] .
\end{aligned}
$$

In particular, for $n=k_{1}-1$, we find the value of $P_{k_{2}-1,1}$

$$
\begin{aligned}
(m- & \left.k_{1}+Y+1\right) B \rho_{1}^{*} P_{k_{1}-1,1} \\
= & D \frac{\left(1+\left(k_{1}-1\right) \gamma^{*}\right)\left(k_{2}-k_{1}-1\right) \gamma^{*}}{\left[m+Y-k_{1}\right]_{\left(k_{2}-k_{1}-1\right)}\left(B \rho_{1}^{*}\right)^{\left(k_{2}-k_{1}-1\right)}} \\
& -B \rho_{2}^{*} \sum_{r=1}^{k_{2}-k_{1}-1} \frac{\left(m-k_{2}+Y+r+2\right)\left(1+\left(k_{1}-1\right) \gamma^{*}\right)_{\left(k_{2}-k_{1}-r-1\right) \gamma^{*}}}{\left[m+Y-k_{1}\right]_{\left(k_{2}-k_{1}-r-1\right)}\left(B \rho_{1}^{*}\right)^{\left(k_{2}-k_{1}-r-1\right)}} P_{k_{2}-r-2,2} \\
& +\sum_{r=1}^{k_{2}-k_{1}-1} \frac{\left(1+\left(k_{1}-1\right) \gamma^{*}\right)_{\left(k_{2}-k_{1}-r\right) \gamma^{*}}}{\left[m+Y-k_{1}\right]_{\left(k_{2}-k_{1}-r-1\right)}\left(B \rho_{1}^{*}\right)^{\left(k_{2}+k_{1}-r-1\right)}} P_{k_{2}-r-1,2}=T_{2} .
\end{aligned}
$$

Now from row $i=m+Y-\left(k_{1}-3\right)$, the value of $P_{k_{1}-2,1}$ is

$$
P_{k_{1}-2,1}=\frac{D_{1}}{\left(m-k_{1}+Y+2\right) B \rho_{1}},
$$

where

$$
D_{1}=\left\{1+\left(k_{1}-2\right) \gamma\right\} P_{k_{1}-1,2}-\left(m-k_{1}+Y+2\right) B \rho_{2} P_{k_{1}-2,2}+T_{2} \frac{1+\left(k_{1}-2\right) \gamma}{\left(m-k_{1}+Y+1\right) B \rho_{1}^{*}} .
$$

From row $i=m+Y-\left(k_{1}-4\right)$, the value of $P_{k_{1}-3,1}$ is

$$
\begin{aligned}
& P_{k_{1}-3,1}=\frac{1}{\left(m-k_{1}+Y+3\right) B \rho_{1}}\left[\left\{1+\left(k_{1}-3\right) \gamma\right\} P_{k_{1}-2,2}-\left(m-k_{1}+Y+3\right) B \rho_{2} P_{k_{1}-3,2}\right. \\
&\left.+\frac{1+\left(k_{1}-3\right) \gamma}{\left(m-k_{1}+Y+2\right) B \rho_{1}} D_{1}\right] .
\end{aligned}
$$


Then we can obtain the formula for the solutions for the rows $m+Y-\left(k_{1}-5\right) \leq i \leq m$, that is, $Y+1 \leq n \leq k_{1}-4$ as

$$
\begin{aligned}
P_{n, 1}=\frac{1}{B \rho_{1}}[ & D_{1} \frac{(1+n \gamma)_{\left(k_{1}-n-2\right) \gamma}}{[m+Y-n-1]_{\left(k_{1}-n-2\right)}\left(\beta \rho_{1}\right)^{\left(k_{1}-n-2\right)}} \\
& -B \rho_{2} \sum_{r-1}^{k_{1}-n-2} \frac{\left(m-k_{1}+Y+r+2\right)(1+n \gamma)_{\left(k_{1}-n-r-2\right) \gamma}}{[m+Y-n-1]_{\left(k_{1}-n-r-2\right)}\left(B \rho_{1}\right)^{\left(k_{1}-n-r-2\right)}} P_{k_{1}-r-2,2} \\
& \left.+\sum_{r-1}^{k_{1}-n-2} \frac{(1+n \gamma)_{\left(k_{1}-n-r-1\right) \gamma}}{[m+Y-n-1]_{\left(k_{1}-n-r-2\right)}\left(B \rho_{1}\right)^{\left(k_{1}-n-r-2\right)}} P_{k_{1}-r-1,2}\right] .
\end{aligned}
$$

In particular for $n=Y+1$, we find the value of $P_{Y+1,1}$

$$
\begin{aligned}
(m-1) B \rho_{1} P_{Y+1,1}= & D_{1} \frac{(1+(Y+1) \gamma)_{\left(k_{1}-Y-3\right) \gamma}}{[m-2]_{\left(k_{1}-Y-3\right)}\left(B \rho_{1}\right)^{\left(k_{1}-Y-3\right)}} \\
& -B \rho_{2} \sum_{r=1}^{k_{1}-Y-3} \frac{\left(m-k_{1}+Y+r+2\right)(1+(Y+1) \gamma)_{\left(k_{1}-Y_{1}-r-3\right) \gamma}}{[m-2]_{\left(k_{1}-Y_{1}-r-3\right)}\left(B \rho_{1}\right)^{\left(k_{1}-Y-r-3\right)}} P_{k_{1}-r-2,2} \\
& +\sum_{r=1}^{k_{1}-Y-3} \frac{(1+(Y+1) \gamma)_{\left(k_{1}-Y-r-2\right) \gamma}}{[m-2]_{\left(k_{1}-Y-r-3\right)}\left(B \rho_{1}\right)^{\left(k_{1}-Y-r-3\right)}} P_{k_{1}-r-1,2}=T_{3} .
\end{aligned}
$$

Now from row $i=m+1$, the value of $P_{Y, 1}$ is

$$
P_{Y, 1}=\frac{D_{2}}{m B \rho_{1}}
$$

where

$$
D_{2}=P_{Y, 1} m B \rho_{1}=(1+Y \gamma) P_{Y+1,2}-m B \rho_{2} P_{Y, 2}+\frac{(1+Y \gamma)}{(m-1) B \rho_{1}} T_{3} .
$$

From row $i=m+2$, the value of $P_{Y-1,1}$ is

$$
P_{Y-1,1}=\frac{1}{m B \rho_{1}}\left[\{1+(Y-1) \gamma\} P_{Y, 2}-m B \rho_{2} P_{Y-1,2}+\frac{\{1+(Y-1) \gamma\}}{m B \rho_{1}} D_{2}\right] .
$$

Then we can obtain the formula for the solutions at the rows $m+3 \leq i \leq m+Y$, that is, $1 \leq n \leq Y-2$ as

$$
\begin{aligned}
P_{n, 1}= & \frac{1}{m B \rho_{1}}\left[D_{2} \frac{(1+n \gamma)(Y-n) \gamma}{\left(m \beta \rho_{1}\right)^{(Y-n)}}-m B P_{2} \sum_{r-1}^{Y-n} \frac{(1+n \gamma)(Y-n-r) \gamma}{\left(m B \rho_{1}\right)^{(Y-n-r)}} P Y-r, 2\right. \\
& \left.+\sum_{r-1}^{Y-n} \frac{(1+n \gamma)(Y-n-r) \gamma}{\left(m B \rho_{1}\right)^{(Y-n-r)}} P Y-r+1,2\right] .
\end{aligned}
$$

In particular for $n=1$, we find the value of $P_{1,1}$. 
At the first row $i=m+Y+1$ we find the value of $P_{0,1}$ as

$$
P_{0,1}=\frac{1}{m \rho_{1}} P_{1,1},
$$

where

$$
\begin{aligned}
& {[L]_{\alpha}=L \cdot(L-1) \cdot(L-2) \cdots(L-\alpha-1),} \\
& (L)_{\alpha}=L \cdot(L+1) \cdot(L+2) \cdots(L+\alpha+1) .
\end{aligned}
$$

Thus we succeed to deduce the first branch probabilities $P_{n, 1}$ in terms of the second branch probabilities $P_{n, 2}$.

Substituting relations from (2.21), (2.23), (2.24), (2.25), (2.28), (2.29), (2.30), (2.32), (2.34), (2.35), (2.37), (2.39), (2.40), and (2.41) in the second relations of (2.7), (2.8), (2.9), (2.10), (2.11), (2.12), (2.13), (2.14), (2.15) and by using the relation of (2.18), the second branch probabilities $P_{n, 2}$ can be obtained in an explicit form. And so the first branch probabilities $P_{n, 1}$ can be obtained in an explicit form too.

3. Measures of effectiveness. The expected number of units in the system and in the queue are

$$
L=\sum_{n=0}^{m+Y} n\left(P_{n, 1}+P_{n, 2}\right), \quad L_{q}=\sum_{n=1}^{m+Y}(n-1)\left(P_{n, 1}+P_{n, 2}\right) .
$$

Also, the probability that there are no units in the system is

$$
P_{0}=P_{0,1}+P_{0,2}
$$

\section{Particular cases}

CASE 4.1 (the queue $H 2 / M / 1 / 2 / 2(\beta, \delta)$ ). Let $Y=0$ and $k_{1}=k_{2}=m$, (i.e., $\mu_{1}=\mu_{2}=$ $\mu$ and $\left.\mu_{3}=0\right)$ in the above relations, we get

$$
\begin{aligned}
P_{0,1} & =\frac{\rho_{2}\left\{1+\gamma-a \beta \rho_{2}(1-\alpha)\right\}}{\rho_{1}(a b+d)}, \quad P_{0,2}=\frac{a \beta \rho_{2}(1-\alpha)}{a b+d}, \\
P_{1,1} & =\frac{2 \rho_{2}\left\{1+\gamma-a \beta \rho_{2}(1-\alpha)\right\}}{a b+d}, \quad P_{1,2}=\frac{2 a \beta \rho_{1} \rho_{2}(1-\alpha)}{a b+d}, \\
P_{2,1} & =\frac{2 \alpha \beta \rho_{1} \rho_{2}}{a b+d}, \quad P_{2,2}=\frac{2 \beta \rho_{1} \rho_{2}(1-\alpha)}{a b+d}, \\
P_{0} & =\frac{1}{\rho_{1}(a b+d)}\left\{\rho_{2}(1+\gamma)+a \beta(1-\alpha)\left(\rho_{1}^{2}-\rho_{2}^{2}\right)\right\}, \\
L & =\frac{2 \rho_{2}}{a b+d}\left\{1+\gamma+a \beta(1-\alpha)\left(\rho_{1}-\rho_{2}\right)+2 \beta \rho_{1}\right\}, \quad L_{q}=\frac{2 \beta \rho_{1} \rho_{2}}{a b+d},
\end{aligned}
$$

where

$$
\begin{aligned}
& a=\frac{(1+\gamma)\left(1+\beta \rho_{1}\right)}{\beta\left\{\beta \rho_{1} \rho_{2}+\alpha \rho_{1}+(1-\alpha) \rho_{2}\right\}}, \\
& b=\beta\left(2 \rho_{2}+1\right)\left\{\alpha \rho_{2}+(1-\alpha) \rho_{1}\right\}-\beta \rho_{2}^{2}\{4 \alpha(1-\beta)+3 \beta-2\}, \\
& d=2 \beta \rho_{1} \rho_{2}\{1-2 \alpha(1-\beta)\}-\rho_{2}(1+\gamma)(2-3 \beta) .
\end{aligned}
$$


Also, the machine availability is

$$
\text { M.A. }=\frac{m-L}{m}=1-\frac{\rho_{2}}{a b+d}\left[1+\gamma+a \beta(1-\alpha)\left(\rho_{1}-\rho_{2}\right)+2 \beta \rho_{1}\right] .
$$

And the operative efficiency is

$$
\text { O.E. }=1-\rho_{0}=1-\frac{1}{\rho_{1}(a b+d)}\left\{\rho_{2}(1+\gamma)+a \beta(1-\alpha)\left(\rho_{1}^{2}-\rho_{2}^{2}\right)\right\} .
$$

CASE 4.2 (the queue $M / M / 1 / m+y / m+y$ ). Let

$$
\begin{gathered}
\beta=1, \quad \delta=0, \quad(\text { i.e., } \gamma=0), \quad \alpha=\frac{1}{2}\left(\text { i.e., } \rho_{1}=\rho_{2}=\rho=\frac{\lambda}{\mu}\right), \\
k_{1}=k_{2}=m \quad\left(\text { i.e., } \mu_{1}=\mu_{2}=\mu, \mu_{3}=0\right) .
\end{gathered}
$$

We obtain the results of Harris [5] when $c=1$.

CASE 4.3 (the queue $M / M / 1 / 2 / 2$ ). Let

$$
\begin{gathered}
\beta=1, \quad \delta=0 \quad(\text { i.e., } \gamma=0), \quad Y=0, \\
\alpha=\frac{1}{2}\left(\text { i.e., } \rho_{1}=\rho_{2}=\rho=\frac{\lambda}{\mu}\right), \quad k_{1}=k_{2}=m=2 .
\end{gathered}
$$

We get

$$
\begin{gathered}
P_{0}=\left(2 \rho^{2}+2 \rho+1\right)^{-1}, \quad L=2 \rho(2 \rho+1)\left(2 \rho^{2}+2 \rho+1\right)^{-1}, \\
L_{q}=2 \rho^{2}\left(2 \rho^{2}+2 \rho+1\right)^{-1} .
\end{gathered}
$$

These results agree with that obtained by Harris [5] and Al-Seedy [4].

\section{REFERENCES}

[1] M. O. Abou-El-Ata, An interarrival hyperexponential machine interference with balking and reneging, Microelectron. Reliab. 32 (1992), no. 9, 1251-1256.

[2] R. O. Al-Seedy, The truncated queues and their measures of effectiveness, Master's thesis, Tanta University, 1984.

[3] _ _ Theory of queues: the general solutions of truncated non-Markovian queues, Ph.D. thesis, Tanta University, 1988.

[4] _ The service Erlangian machine interference with balking, Microelectron. Reliab. 32 (1992), no. 5, 705-710.

[5] D. Gross and C. M. Harris, Fundamentals of Queueing Theory, Wiley Series in Probability and Mathematical Statistics, John Wiley \& Sons, New York, 1974. MR 51\#7044. Zbl 312.60046.

[6] S. K. Gupta, Queues with hyper-Poisson input and exponential service time distribution with state dependent arrival and service rates, Oper. Res. 15 (1967), 847-856. Zbl 178.20803.

Ragab Omarah Al-SeEdy: Department of Mathematics, Teacher's College in Al-AhSA, SAUDI ARABIA

Fawziah M. Al-Ibraheem: Department of Mathematics, Science College For Girls in DAMMAM, SAUDI ARABIA 


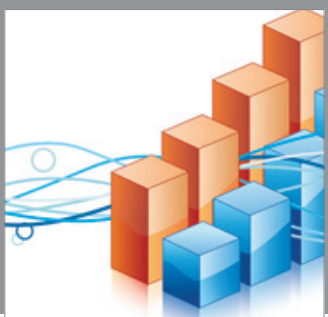

Advances in

Operations Research

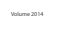

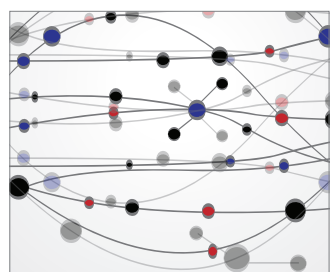

\section{The Scientific} World Journal
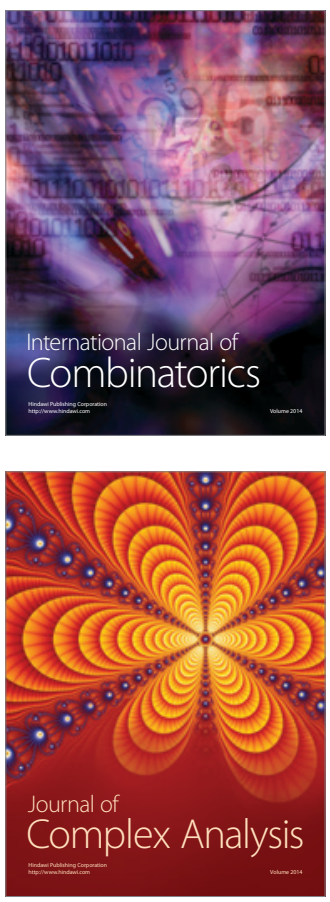

International Journal of

Mathematics and

Mathematical

Sciences
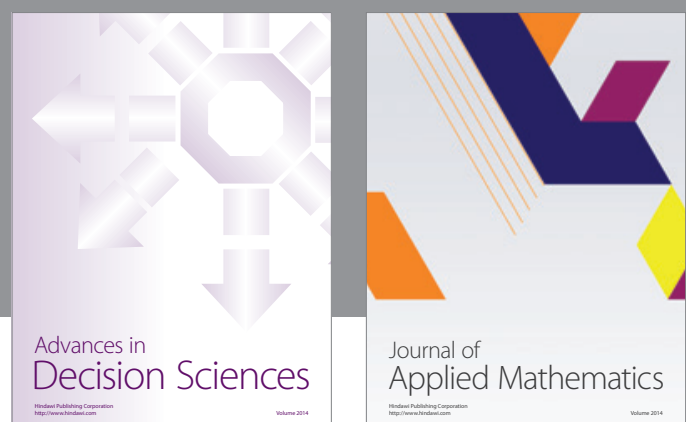

Journal of

Applied Mathematics
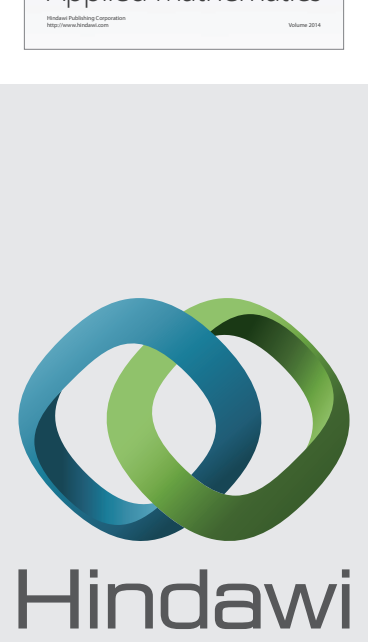

Submit your manuscripts at http://www.hindawi.com
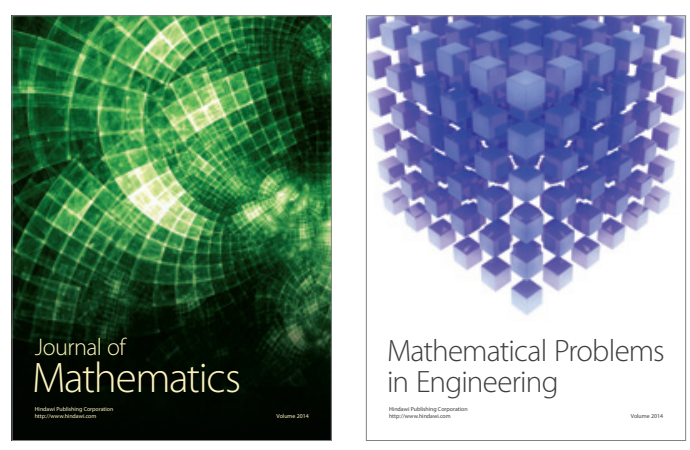

Mathematical Problems in Engineering
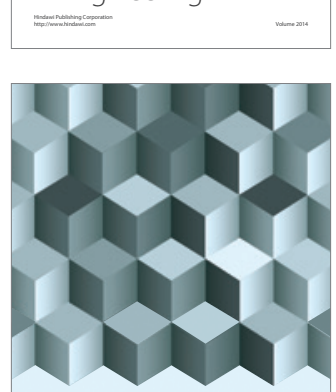

Journal of

Function Spaces
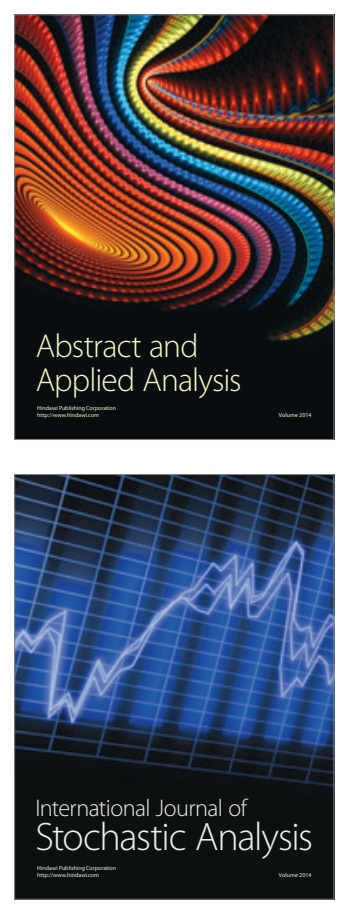

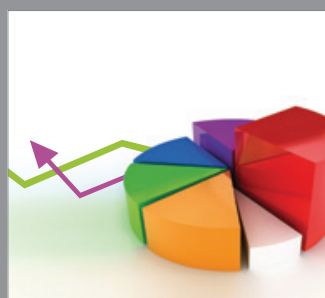

ournal of

Probability and Statistics

Promensencen
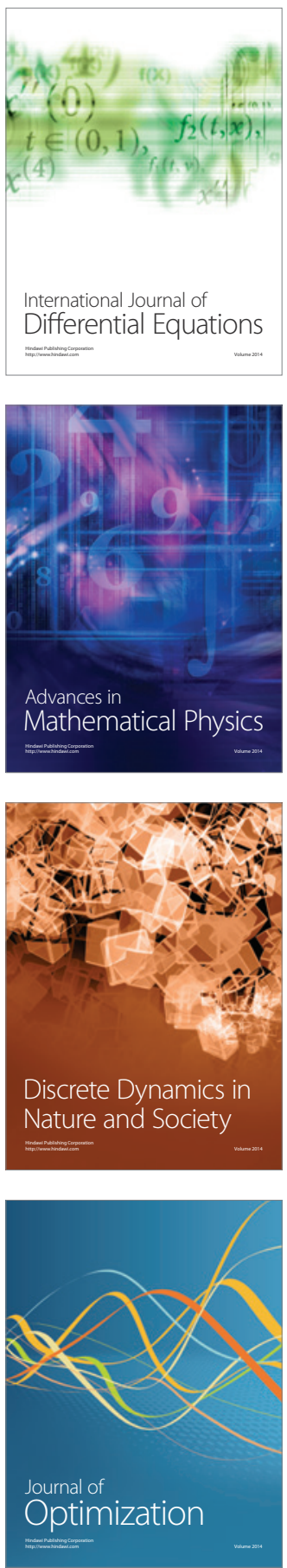